\title{
Dibekacina en el tratamiento de infecciones del tracto urinario en niños. Eficacia y Farmacocinética
}

\author{
Valeria Prado; $;^{1 \cdot 2}$ Loreto Pena; $;^{2}$ Soledad Tagle; ${ }^{2}$ Antonio Banfi, ${ }^{2}$ y T.M. Ximena Aguirre. ${ }^{3}$
}

DIBEKACIN IN URINARY TRACT INFECTIONS OF CHILDREN. EFFICACY AND FARMACOKINETIC

\begin{abstract}
Twenty inpatients with UTI (Urimary tract inlections) mainly callsed by unusual organisms were studied. These putients were treated with Dibekacin: $3 \mathrm{mg} / \mathrm{kg} / \mathrm{day}$ in two intranuscular doses for ten days. Clinical and bucteriological parameters were measured.

In infants all samples were taken by suprapubic vesical aspiration, in children the method employed was a inid strean collection.

In five patients plasma concentrations of Dilsekacin were detennined by the micromethod of Simon and Ying using $\mathbf{B}$. Subtilis as test organism; according to this data, half-iile of Dibekacin was calculated.

Clinical-bacteriological improvertent was achieved in 70\% of panents with UTT. The highest Dibekacin senum concentration was olserved at 0.5 hours $(3.95 \pm 0.79)$ and $\mathrm{T} / 2$ was 3.39 hours.
\end{abstract}

En nuestro medio hemos observado con profunda inquietud que bacterias Gram negativas como E. coli, Klebsiella, Enterobacter, Pseudomonas y Salmonella typhimurium han ido adquiriendo resistencia a los aminoglicósidos, alcanzando cifras verdaderamente alarmantes. Esto cobra mayor importancia en las cepas de origen intrahospitalario.

Considerando que uno de los mecanismos de resistencia de las bacterias Gram negativas frente a aminoglicósidos, se basa en la acción de enzimas que actúan a nivel de los grupos hidróxilos, provocando acetilación, investigadores japoneses, ${ }^{1}$ han sintetizado un aminoglicósido derivado de la Kanamicina, la Dibekacina (3', 4', dideoxykanamicina B) que no posee grupos hidróxilos, lo que permitiría evitar la inactivación enzimática. Investigaciones en cobayos han demostrado que la ototoxicidad de la Dibekacina es menor que la de la Centamicina. ${ }^{2}$

La actividad in vitro de este aminoglicósido frente a cepas aisladas en nuestro ambiente, ha sido determinado por el método de Kirby-Bauer, observándose una actividad superior a la de Gentamicina y similar a la de Amikacina. ${ }^{3}$

\footnotetext{
1 Médico. Unidad de Microbiología. División Ciencias Médicas Oriente, Facultad de Medicina, Universidad de Chile.

2.Médica. Servicio Pediatria, Hospital Luis Calvo Mackenna, Santiago, Chite.

${ }^{3}$ Tecnólogo Medico. Unidad de Micrubiologia. División Ciencias Médicas Otiente, Facultad de Medicina, Universidad de Chile.
}

Tomando en consideración los antecedentes expuestos, el propósito de esta investigación fue evaluar la eficacia tanto clínica como bacteriológica de la Dibekacina en el tratamiento de infecciones del tracto urinario en niños.

Además, en cinco pacientes, se planteo efectuar un análisis farmacocinético de las concentraciones séricas que la Dibekacina alcanza en niños.

\section{MATERIAL Y METODOS}

Se estudiaron 20 pacientes hospitalizados en el Servicio de Pediatría del Hospital Luis Calvo Mackenna que presentaban Infección Urinaria; 10 tenian infecciones por E. coli, 7 por Klebsiella, 2 por Enterobacter y 1 por Citrobacter.

a) En diecisiete de estos niños se trataba del primer episodio de Infección Urinaria, en dos de ellos había fracasado el tratamiento previo con Gentamicina. b) Los tres restantes tenían malformaciones urologicas comprobadas y antecedentes de Infecciones Urinarias repetidas. c) El total de pacientes se distribuía en tres escolares, dos preescolares y quince lactantes.

En todos los lactantes la toma de muestras se efectuó mediante punción vesical suprapúbica. En los escolares se recolectó orina miccional de segundo chorro, en forma aséptica.

A cada paciente se le administró Dibekacina sulfato (Dibe M, Lab. Beta), en dosis de 3 mgxkgxdia, dividida cada doce horas por via intramuscular durante diez días. 
Con el objeto de valorar la curación bacteriológica se efectuaron urocultivos con recuento de colonias de control, entre el cuarto y sexto día de tratamiento y $\mathbf{2 4}$ a $\mathbf{4 8}$ horas después de finalizado. La respuesta observada se catalogó de acuerdo a la siguiente pauta de evaluación como:

a) Muy Buena: Utocultivo de control al $4^{\circ}-6^{\circ}$ día negativo y urocultivo post-tratamiento negativo.

b) Satisfactoria: Urocultivo positivo al $4^{\circ}-6^{\circ}$ dia y negativo post-tratamiento.

c) Mala: Urocultivos de control al $4^{0}$ - $6^{\circ}$ día y post-tratamiento positivos, o agravamiento de las condiciones generales que obligaron a cambiar tratamiento precozmente.

Se efectuaron los siguientes bioanálisis pre y post-tratamiento: Hemograma, Nitrógeno ureico, Velocidad de eritrosedimentación.

En cada cepa aislada se estudió la sensibilidad in vitro mediante método de difusión (KirbyBauer) frente a los signientes antinicrubianos: Amikacina, Dibekacina, Gentamicina, Cotrinıoxazol, Cloramienicol, Ampicilina y Nitrofurantóna. Además se determinó la concentración inhibitoria mínima (CIM) por el método de dilución en placa de Dibekacina, Gentamicina y Arnikacina frente a las mismas cepas.

En cinco pacientes se determinaron las concentraciones plasmáticas de Dibekacina, mediante el micrométodo biológico de Simon y Yin, ${ }^{4}$ utilizando como cepa standard Bacillus subtilis ATCC 6633 y tampón fosfato $\mathrm{pH} 8$ para la dilución del antibiótico y las muestras de sangre.

A cada paciente le fueron tomadas muestras de aproximadamente $0.5 \mathrm{cc}$ de sangre mediante micropunciones del pulpejo o talón con los siguientes intervalos: $0-1 / 2-1-4$ y 6 horas después de la primera administración de Dibekacina intramuscular.

Para el análisis farmacocinético se utilizó el modelo de un compartimiento abierto y a partir de los niveles séricos se detenninó la vida media, T/2 que se calculó suponiendo que a los 30 minutos el proceso de absorción habia concluido.

Las concentraciones de Dibekacina en los diferentes momentos entre 0.5 y 6 horas caen en una recta: La vida media y la constante de eliminación (Ke) se calcularon de la pendiente de dicha recta ajustada por el método de los cuadrados minimos.

Se utilizó para los cálculos las siguientes ecuaciones: a) $\mathrm{C}=\mathrm{C}_{0} \mathrm{e}^{-\mathrm{kt}}$, en la cual $\mathrm{C}$ es la concentración plasmática en el tiempo $t$, y $K$ la constante de velocidad de eliminación.

b) $\log \mathrm{C}=\log \mathrm{C}_{0}-\frac{\mathrm{K}}{2.3} \mathrm{t}$

Esta expresión maternática corresponde a una recta de cuya pendiente puede obtenerse $K$ y del intercepto la concentración in icial $\mathrm{C}_{\mathrm{v}}$.

En estos cinco pacientes, se estudió la capacidad bactericida del suero, de la muestra tomada a los 30 minutos de la administración del antibiótico, frente a la cepa que provocaba la infección, determinándosse el título de inhibición.

Además, se cuantificó de la misma manera, la capacidad inhibitoria de la orina, tomando una alícuota del volumen recolectado despues de las doce horas posteriores a la primera dosis de Dibekacina.

\section{RESULTADOS}

La Tabla 1 muestra la distribución por edad y sexo de los 20 pacientes con Infección Urinaria trata‘os con Dibekacina. Predominan los lactantes menores de 12 meses y el sexo masculino.

Tabla 1

Distribución por edad y sexo de 20 pacientes con infección urinaria tratados con dibekacina

\begin{tabular}{lccc}
\hline Ediad & $\begin{array}{c}\text { N. de pa- } \\
\text { cientes }\end{array}$ & \multicolumn{2}{c}{ Sexo } \\
& & Masculino Femenino \\
\hline 0-3 meses & 8 & 8 & 0 \\
3 ineses 1 dia-12 meses & 7 & 4 & 3 \\
1 año-6 años & 2 & 0 & 2 \\
6 años 1 día-13 años & 3 & 1 & 2 \\
Total & 20 & 13 & 7 \\
\hline
\end{tabular}

Tabla 2

Etiología bacteriana de infección urinaria en 20 pacientes tratados con dibekacina

\begin{tabular}{lcc}
\hline Bacterias & Aisladas N. & Erradicadas N. \\
E. Coli & 10 & 9 \\
$\quad$ Klebsiella & 7 & 4 \\
Enterobacter & 2 & 1 \\
Citrobacter & 1 & 1 \\
\hline Tutal & 20 & 15 \\
\hline
\end{tabular}

En la Tabla 2 aparecen detalladas las bacterias aisladas en los 20 pacientes con Infección Urina- 
Tabla 3

Sensibilidad in vitro (Kirby Bauer) de enterobacterias aisladas de 20 pacientes con 1TU* frente a 7 antimicrobianus

\begin{tabular}{|c|c|c|c|c|c|c|c|}
\hline \multirow[t]{2}{*}{ Antimicrobiano } & \multicolumn{5}{|c|}{ Número de cepas serısibles } & \multicolumn{2}{|c|}{ Total cepas sensibles } \\
\hline & E. & Coli & Klebsiella & Enterobacter & Gitrobacter & $N .0$ & $\%$ \\
\hline Dibekacina & & $9 / 10$ & 37 & $0 / 2$ & $0 / 1$ & 12 & 60 \\
\hline Amikacina & & $9 / 10$ & $6 / 7$ & $2 / 2$ & $0 / 1$ & 17 & 85 \\
\hline Gentamicina & & $6 / 10$ & $2 / 7$ & $0 / 2$ & $0 / 1$ & 8 & 40 \\
\hline Cotrimoxazol & & $8 / 10$ & $4 / 7$ & $0 / 2$ & $0 / 2$ & 12 & 60 \\
\hline Cloramfenicol & & $3 / 10$ & $1 / 7$ & $0 / 2$ & $0 / 1$ & 4 & 20 \\
\hline Ampicilina & & $0 / 10$ & $1 / 7$ & $0 / 2$ & $0 / 1$ & 1 & 5 \\
\hline Nitrofiurantóna & & $6 / 10$ & $1 / 7$ & $1 / 2$ & $0 / 1$ & 8 & 40 \\
\hline
\end{tabular}

*ITU: Infecrión del tracto urinario.

\section{Tabla 4}

CIM en $\mathrm{mg} x \mathrm{ml}$ de dibekacina, amikacina $y$ gentamicina frente a enterobacterias aisladas de pacientes con ITU*

\begin{tabular}{|c|c|c|c|}
\hline & $\begin{array}{c}\text { E. Coli } \\
\text { (10 Cepas) }\end{array}$ & $\begin{array}{l}\text { Klebsiella } \\
\text { (7 Cepas) }\end{array}$ & $\begin{array}{c}\text { Enterobacter } \\
\text { (2 Cepas) }\end{array}$ \\
\hline \multicolumn{4}{|l|}{ Dibekacina } \\
\hline $\mathrm{X} \pm \mathrm{DS}$ & $15.31 \pm 29.9$ & $34.9 \pm 34.8$ & 75.0 \\
\hline Moda & 6.25 & 50.0 & --- \\
\hline Cepas semsibles & $9 / 10$ & $3 / 7$ & --- \\
\hline \multicolumn{4}{|l|}{ Amikacina } \\
\hline $\mathrm{X} \pm \mathrm{DS}$ & $3.43 \pm 7.6$ & $1.5 \pm 2.1 .3$ & 0,39 \\
\hline Moda & 0,78 & 0.39 & --- \\
\hline Cepas sensibles & $9 / 10$ & $7 / 7$ & --- \\
\hline \multicolumn{4}{|l|}{ Gentamicina } \\
\hline $\mathrm{x} \pm \mathrm{DS}$ & $56.59 \pm 49.0$ & $57.39 \pm 53.14$ & 100.0 \\
\hline Moda & 100.0 & 100.0 & --- \\
\hline Cepas sensibles & $5 / 10$ & $3 / 7$ & --- \\
\hline
\end{tabular}

*ITU: Infección del tracto urinario

ria, de las cuales el $75 \%$ fueron erradicadas con el tratamiento $(15 / 20)$.

En la tabla 3 tenemos los resultados del estudio de sensibilidad in vitro de las cepas aisladas frente a 7 antimicrobianos por el niétodode Kirby Bauer. Dibekacina resultó activa frente al $60 \%$ de las cepas.

La Tabla 4 registra los promedios de CIM en $\mathrm{Mg} \times \mathrm{ml}$ de Dibekacina, Amikacina y Gentamicina, a las cepas aisladis.

En la Tabla 5 vemos la respuesta bacteriológica al tratamiento con Dibekacina de los 20 pacientes con Infección Urinaria, catalogada según pauta preestablecida, obteniéndose resultados satisfactorios en el $70 \%$ de los casos.
Tabla 5

Respuesta clínico-bacteriológica de 20 pacientes con infección uninaria tratados con dibekacina

\begin{tabular}{lcc}
\hline Respuesta & N. ${ }^{\circ}$ de pacientes & $\%$ \\
Muy buena & 11 & 55 \\
Satisfactoria & 3 & 15 \\
Mala & $6^{*}$ & 30 \\
Total & 20 & 100
\end{tabular}


Factores presentes en 6 pacientes con infección urinaria con fracaso terapéutico a dibekacina

\begin{tabular}{llcccc}
\hline Paciente & $\begin{array}{l}\text { Agente } \\
\text { Etiologico }\end{array}$ & $\begin{array}{l}\text { Fracaso Previo } \\
\text { con Gentamicinà }\end{array}$ & $\begin{array}{l}\text { Cepa Resistente Malformación Desnutrición } \\
\text { a Dibekacina }\end{array}$ & $\begin{array}{c}\text { Prematuridad } \\
\text { Urulógica }\end{array}$ \\
\hline G. JS & Klebsiella & $*$ & $*$ & $*$ & \\
2. CSP & Enterobacter & $*$ & $*$ & $*$ & $*$ \\
3. CSS & E. Coli & $*$ & $*$ & $*$ \\
4. PH (1) & E. Coli & $*$ & $*$ & $*$ \\
5. CC & Klebsiella & $*$ & $*$ & & $*$ \\
6. JM & Klebsiella & & & & $*$ \\
\hline
\end{tabular}

(1) Superinfección por Klebsiella

Tabla 7

Concentraciones Plasmáticus de Dibekacina en nin̉os vía intramuscular, dosis $1.5 \mathrm{rng} / \mathrm{kg}$ peso.

\begin{tabular}{|c|c|c|c|c|c|c|c|}
\hline \multirow[t]{2}{*}{ Paciente } & \multirow[t]{2}{*}{ Edad } & \multirow[t]{2}{*}{ Peso KG. } & \multicolumn{5}{|c|}{ Concentraciones Séricas MCG/ML } \\
\hline & & & O HR. & $0.5 \mathrm{HR}$. & $1 \mathrm{HR}$. & $4 \mathrm{HR}$. & $6 \mathrm{HR}$. \\
\hline J.S. & $2 \mathrm{~m}$ & 3.27 & 0.0 & 2,40 & 2.50 & 0.60 & --- \\
\hline M.M. & $2 m$ & 4.20 & 0.0 & 4.45 & 1.85 & --- & 0.35 \\
\hline R.O. & $6 \mathrm{~m}$ & 4.95 & 0.0 & 5.10 & 3.00 & 2.58 & 2.15 \\
\hline M.R. & $13 a$ & 29.40 & 0.0 & 3.40 & 2.50 & 1.98 & 1.45 \\
\hline R.D. & 9 a & 27.40 & 1.1 & 3.40 & 5.50 & 2,00 & 0.88 \\
\hline $\begin{array}{l}\bar{X} \pm \\
\text { DS }\end{array}$ & & & & $\begin{array}{c}3.95 \pm \\
0.79\end{array}$ & $\begin{array}{c}3.07 \pm \\
1.42\end{array}$ & $\begin{array}{c}1.79 \pm \\
0.84\end{array}$ & $\begin{array}{c}1.20 \pm \\
0.77\end{array}$ \\
\hline
\end{tabular}

Vida Media T $1 / 2=3.39$ HRS.

Gráfico 1 = Curva de Niveles Plasmáticos

$$
K_{e}=0.2043
$$

La Tabla 6 analiza algunos factores presentes en los pacientes en los cuales tracasó el tratamiento con Dibekacina.

En la Tabla 7 están expresadas las concentraciones plasmáticas de Dibekacina, obtenidas en 5 pacientes despues de la administración de $1.5 \mathrm{mg} / \mathrm{kg}$-p por vía intramuscular. De acuerdo a estos valores la vida media calculada fue de 3.39 horas.

El Gráfico 1 muestra la curva de eliminación de Dibekacina a partir de la 0.5 hora, que cae en una recta cuya pendiente fue ajustada por el método de los cuadrados mínimos.

En la Tabla 8 se expresa la capacidad inhibitoria del plasma, a los 30 minutos de administrada la Dibekacina frente a la cepa que causaba la infección y la capacidad inhibitoria de una alícuota de la orina recolectada durante las doce horas posteriores a la primera dosis del antibiótico. La correlación con la respuesta observada muestra que títulos superiores a $1 / 8$ en orina significan curación.

\section{Tabla 8}

Correlación entre capacidad de inhibición plasnuática e inhibición urinaria y respuesta clínico-bacteriológica en 5 pacientes con infección urinaria

\begin{tabular}{|c|c|c|c|}
\hline Paciente & $\begin{array}{c}\text { Título inhibi- } \\
\text { ción plasmáti- } \\
\text { ca } \\
\text { plasma }=0.5 \mathrm{~h}\end{array}$ & $\begin{array}{l}\text { Título inhibi- } \\
\text { ción urinaria } \\
\text { x orina } \mathbf{1 2} \text { h }\end{array}$ & $\begin{array}{l}\text { Respuesta cli- } \\
\text { nicos bacterio- } \\
\text { lógica }\end{array}$ \\
\hline J.S. & $\mathbf{R}$ & $\mathbf{F}$ & Mala \\
\hline M.M & $1 / 4$ & $1 / 8$ & Muy buena \\
\hline R.O. & $1 / 4$ & $1 / 8$ & Muy buena \\
\hline M.R. & $1 / 4$ & $1 / 8$ & Muy buena \\
\hline R.D & $\mathbf{F}$ & $\mathbf{H}$ & Muy buena \\
\hline
\end{tabular}




\section{Niveles plasmaticos de DIBEKACINA despues de administración I.M.}

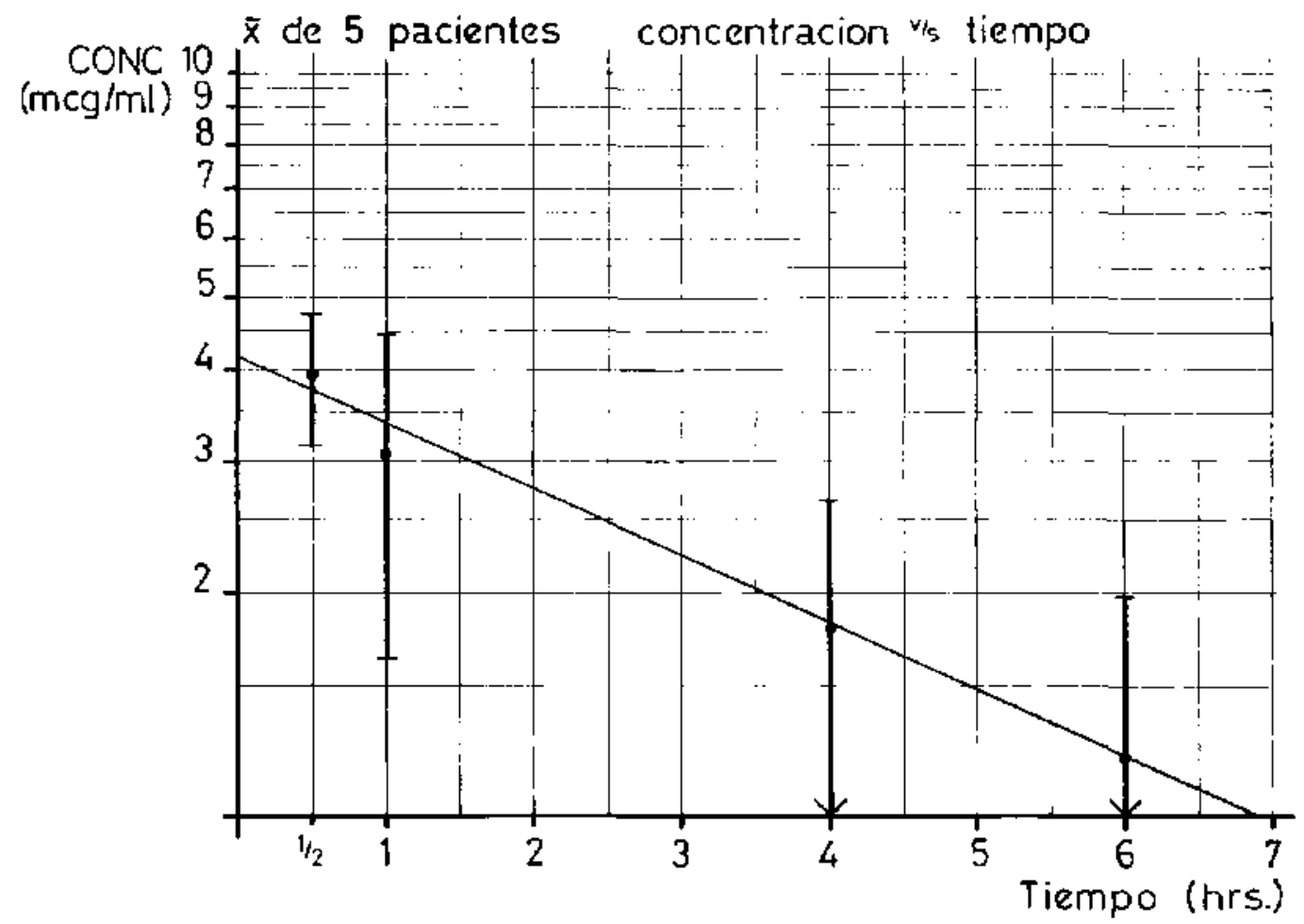

En la Tabla 9 observanos los promedius de los valores de Hemoglobina y N. Ureico antes y despues del tratamiento con Dibekacina, los cuales no muestran diferencias significativas. Lo mismo los valores de la eritrosedimentación.

Tabla 9

Parímetros bioquímicos en 20 pacietites con infección urinaria tratados con Dibekacina

\begin{tabular}{|c|c|c|}
\hline & Pre-tratamiento & Post-tratamiento \\
\hline & $\begin{array}{ll}\overline{\mathbf{x}} & \text { ISS }\end{array}$ & $\bar{x} \quad$ DS \\
\hline Нетnoglohina & $11.75 \pm 1.84$ & $10.84 \pm 2.12$ \\
\hline N. U Ueico & $11.07 \pm 3.66$ & $11.80 \pm 4.38$ \\
\hline$V \mathrm{HS}$ & $26.27=28.38$ & $17.27 \pm 13.58$ \\
\hline
\end{tabular}

\section{COMENTARIOS}

El grupo de pacientes incluidos en nuestro estudio constituia una muestra seleccionada, tanto por las características de los pacientes (edad y sexo: Cuadro 1) como por las agentes causales de infección urinaria, cuya distribución no es la habitual considerando que la mayora de los casos ( 17 de 20) correspondía a un primer episodio de Infección Urinaria.

La elevada resistencia de estas cepas frente a los antimicrobianos señala que constituían verdadero desafío terapéutico (Cuadros 3 y 4). Además en 5 de estos pacientes con Infección Urinaria por Klebsiella, estas cepas fueron adquiridas dentro del ambiente hospitalario. Correlacionando los resultados del estudio de sensibilidad in vitro con la respuesta clínico-bacteriológica, observamos que la actividad in vivo de Dibekacina fue superior a la observada en el laboratorio (Cuadros $3,4,5$ ). Considerando estos aspectos, estimados que el $70 \%$ de eficacia obtenida con Dibekacina, es satisfactoria, evaluado esto de acuerdo a los criterios de curación bacteriológica preestablecidos. Si nos remitimos solamente a los pacientes 
con bacterias sensibles a la Dibekacina, el éxito terapéntico en este grupo fue del $100 \%$.

En 6 pacientes en los cuales el tratamiento con Dibekacina fracasó, es interesante analizar factores del huésped y de los gérmenes: cinco de ellos presentaban Infección Uninaria por bacterias resistentes a Dibekacina cepas que además eran resistentes a otrus 6 antibióticos; en 4 de ellos habia fracasado el tratamiento previo con Gentamicina. De estos niños, 2 tenían además malformaciones de la vía urinaria y otros dos pacientes eran niños de pre-término con pesos de $1.890 \mathrm{y}$ $2.070 \mathrm{grs}$. en el mornento de la Infección Urinaria. Un paciente prematuro con infección por $\mathrm{E}$. coli, sensible a Dibekacina, era un desnutrido grave, con un déficit ponderal de $30 \%$ y una cardiopatía congénita compleja, que sufrió una sobreinfección urinaria por Klebsiella.

En cuanto a los promedios de las concentraciones plasmáticas de Dibekacina obtenidos, después de la administración de una primera dosis de $1.5 \mathrm{mg} / \mathrm{kg}$ IM, éstos resultaron inferiores a $10 \mathrm{~s}$ valores obtenidos en adultos, con dosis similares, por otros investigadores, ${ }^{5,6}$ lo que podría explicarse porque dependen del volumen de distribución y éste es superior en los niños, que tienen mayor proporción de agua corporal que los adultos.

Otro aspecto que considerar, es que nosotros utilizamos en nuestros pacientes el micrométodo de Simon y Yin; los datos de los trabajos antes citados fueron obtenidos utilizando otra técnica, aunque la cepa standard en ambos casos es la misma: Bacillus subtilis ATCC 6633.

Seria interesante, en estudios posteriores, efectuar determinaciones de niveles plasmáticos con dosis mayor de Dibekacina, ya que considerando la CIM de las cepas, resultaría conveniente tratar de obtener concentraciones más elevadas en plasma puesto que ellas son proporcionales a la dosis, ${ }^{5}$ evaluando los efectos adversos.

La vida media de Dibekacina en nuestros niños (3.39 hrs.), es superior y casi el doble de la obtenida en pacientes adultos con dosis equivalentes, ${ }^{5}$ esto se debería a que está influida principalmente por la depuración y no por los niveles plasmáticos.

Queremos destacar una información que resulta de mucha ayuda para un manejo clínico más racional de pacientes sometidos a terapia antimicrobiana, cual es la detenninación de la capacidad bacteriana del plasma y en este caso de la orina, del paciente frente a la cepa que está causando la infección; esto se puede cuantificar y constituye la curva de inhibición plasmática e inhibición urinaria respectivamente. ${ }^{7,8}$ En general se considera que un título superior a l/8 asegura un éxito terapéutico, esto se vio corroborado en 4 de los 5 pacientes en los cuales se hizo esta deterninación: tres pacientes con título de inhibición plasmática $1 / 4$ inhibición urinaria 1/8 tuvieron muy buena respuesta clínico-bacteriológica, 1 paciente con resistencia plasmática y urinaria resultó fracaso terapéutico. El título inhibitorio de la orina es superior al plasmático debido a que Dibekacina alcanza concentraciones 10 veces superior en orina. ${ }^{6,9}$

En una paciente no concordó este parámetro ya que tenía resistencia plassnática y urinaria, una infección causada por una cepa de Enterobacter cloacae, con una concentración inhibitoria minima (CIM) de Dibekacina de $100 \mathrm{mog} / \mathrm{ml}$, con gran sintomatología clinica y sin embargo huvo una excelente respuesta clínco-bacteriológica.

En esta paciente, una escolar de 9 años, habría que atribuji su curación principalmente a una buena respuesta inmune y apoyaria esta hipótesis el hecho de que en ella, no habiendo recibido ningún antibiótico previamente, la muestra de plasma obtenida al tiempo 0 , mostró $1.1 \mathrm{mcg} / \mathrm{ml}$ de actividad antibiótica. Como se utilizô un método microbiológico, esto correspondería a una expresión de la acción bactericida normal del suero de esta paciente probablemente mediada por la acción del complemento frente a la cepa Bacillus subtilis. Ha sido demostrado que esta capacidad bactericida del complemento es muy eficiente frente a muchas bacterias Gram negativas. ${ }^{10}$

Comparaciones del hemograma, orina sedimento y $\mathrm{N}$. ureico, pre y post-tratamiento no mostraron efectos tóxicos medulares ni a nivel renal, en estos pacientes. Auncyue reconocemos que para detectar compromiso renal con mayor exactitud, sería necesario efechuar determinaciones de enzimas urinarias como la $\mathrm{N}$-acetil BD glucosaminidasa, fosfatasas alcalinas y alanina aminopeptidasa, análisis todos ellos que están fuera de nuestras posibilidades; solo podemos decir que con los controles practicados no observamos toxicidad renal.

\section{CONCLUSIONES}

En el grupo de 20 pacientes con Infecciön Urinaria estudiados, que representaba un desafio terapéutico (edad, malformaciones urológrićas y ce- 
pas multirresistentes), se obtuvo un $70 \%$ de curación clínico-bacteriológica con Dibekacina.

El estudio de sensibilidad in vitro efectuado, indicaría que la Dibekacina es más activa que Gentamicina frente a gérmenes Gram negabvos aislados de infecciones urinarias y que Amikacina podría ser superior frente a algunas cepas de Klebsiella.

La farmacocinética de Dibekacina en nin̄os, mostró concentraciones plasmáticas inferiores a las observadas en adultos, con una vida media superior.

No se observaron efectos adversos con el uso de este antimicrobiano.

\section{AGRADECIMIENTOS}

Nuestros sinceros agradecimientos al Dr. Aquiles Arancibia, por su asesoría técnica en los cálculos farmacocinéticos.

\section{RESUMEN}

Se estudiaron 20 pacientes con Infecciones Urinarias causadas principalmente por bacterias poco frecuentes en esta patología. Estos pacientes recibieron Dibekacina sulfato $3 \mathrm{mg} / \mathrm{kg}$-p dia, en 2 dosis, por via IM durante 10 dias. Se evaluaron en ellos parámetros clínicos y bacteriológicos.

En los lactantes las muestras de orina fueron recogidas mediante punción vesical suprapúbica y en los escolares se tomó orina miccional de segundo chorro.
En 5 pacientes se determinaron las concentraciones plasmaticas de Dibekacina por el micrométodo biológico de Simon y Yin.

Se obtuvo mejoría clínico-bacteriológica en el $70 \%$ de los pacientes. La concentración plasmática más elevada de Dibekacina se observó a las 0.5 hr. $(3.95 \pm 0.79)$ y el T/2 calculado fue de 3,39 .

\section{REFERENCLAS}

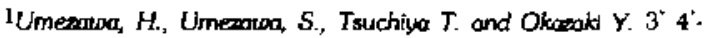
dideoxylanamycin $B$ active against kanamycin resistant $\mathrm{E}$. coli and P. aeruginosa. J. Antibiotics $24(7)$ 485-487, 1971.

${ }^{2}$ Nakaseswo T., Fukushima K. Auditory effects of a new antibiotic 3', 4'-dideoxykanamyein B in guinea pigs. Japón. J. Artibiot. $26 \mathrm{~N}: 1,24-27,1973$.

3Montiel, F. Laboratorio de Microbiología del Hospital de la Universidad Católica Santiago. Comunicación personal, 1980.

4 Simon H. J. and Yin E. J. Microbipassuy of antimicrobial agents. App. Microbiol. 19:573.579, 1969.

5 Umemurr $K$, Komlya I. et al. Phurnacokinetics of Dibekacin after intramuscular administration in man. Japón. J. Antibiot. $30(9): 650-657,1977$.

${ }^{6}$ Sowae $Y$, Told $M$. and Hata Y. Pharmacokinetics of Dibekacin and its clinical efficacy after intravenous drip infusion. Japón. J. Antibiot. 30 (9); $667-671,1977$

TPlen F. D. Vost K. L. Variation in perfomance of the serum bactericidal test. Antimicrob. Ag. Chemother. Vol. $6 \mathrm{~N}^{\circ} 3$, 330-333, 1974.

8 dauct $E$ Assay of antibacterial activity in serum. Amer. J. Dis. Child. 103: 113-116, 1962.

${ }^{9}$ Fuj, $R$, Hashire S. Dibekacin in children: clinical evaluation and phambacokinetics. Current Chemotherapy $933-935,1978$.

10Ruddy, S., Gigito, I. and Austen K. F. The complement system of man (four parts) N. Engl. J. Med. 287: 489-495, 598-596, 642646. 1972 . 